\title{
Determination of fiber-structure for the effective reinforcement of planar composite-material constructions
}

\author{
Natalia Feodorova \\ Siberian Federal University, \\ Svobodny 79, Krasnoyarsk, 660041, Russia \\ email nfeodorova@sfu-kras.ru, feodorova.natalia@mail.ru
}

Keywords: reinforcement, structural model, curvilinear trajectories, cracking resistance, breaking strains.

\begin{abstract}
To describe the behaviour of a composite material reinforced by fibers along curvilinear trajectories, a resolving system of differential equations has been obtained. I developed a structural model within the framework of the two-dimensional non-uniform linear elasticity problem. The reinforcement is performed on the basis of three approaches: according to the grid lines of the orthogonal coordinate system determined by the given conformal representation; according to trajectories isogonal to the given curves; according to the helical trajectories in the axisymmetric formulation of the problem. Particular solutions of the corresponding boundary problems have been obtained. Recommendations for the effective reinforcement of planar constructions have been presented.
\end{abstract}

\section{Introduction}

Thin-walled elements produced by introducing fibrous composite materials have been widely used in modern aerospace and machine-building industry. Fibrous reinforcement allows applying new principles in product design and production, the material and product being created simultaneously as a common integrated technological process. As a result, a product with new unique performance qualities is obtained [1,2,3,4,5,6,7]. Until recently, reinforcement has been carried out predominantly using rectilinear fiber-structures. Such reinforcement structures may be inefficient for constructions with high gradients of the stress and strain fields in the area of apertures and transitional elements. In this case, it is necessary to create constructions with special curved reinforcement structures. Recent literature $[8,9,10,11,12]$ and the present paper focus on the methods of finding such reinforcement structures.

\section{Statement of the axisymmetric problem of reinforced media}

The stress-strain state of a reinforced plate in the polar coordinate system $(\rho, \theta)$ relative to the components of the strain $\varepsilon_{\rho}, \varepsilon_{\theta}, \varepsilon_{\rho \theta}$ and stress $\sigma_{\rho}, \sigma_{\theta}, \sigma_{\rho \theta}$ tensors in the axisymmetric case (the functions sought are independent of the polar angle $\theta$ ) is described by relations (1) - (5) below. The equilibrium equations have the following form

$$
\frac{d \sigma_{\rho}}{d \rho}+\frac{\sigma_{\rho}-\sigma_{\theta}}{\rho}=0, \frac{d \sigma_{\rho \theta}}{d \rho}+\frac{2}{\rho} \sigma_{\rho \theta}=0 .
$$

Let the reinforcement be performed by $m$ classes of the fibers $(m=1,2,3), \varphi_{m}-$ are the reinforcement angles, $\varepsilon_{m}$ - is the deformation in the fiber, $\sigma_{m}-$ is the stress in the fiber, $\omega_{m}-$ is the power of reinforcement by the $m$-th class of fibers. We determine the deformations in the fiber in the polar system using structural model [13]

$$
\varepsilon_{\rho} \cos ^{2} \varphi_{m}+\varepsilon_{\theta} \sin ^{2} \varphi_{m}+\varepsilon_{\rho \theta} \cos \varphi_{m} \sin \varphi_{m}=\varepsilon_{m}
$$


The Cauchy relations connecting the components of the deformation tensor and those of the shear vector $u_{\rho}, u_{\theta}$, in the conditions of axisymmetric deformation have the form

$$
\varepsilon_{\rho}=\frac{d u_{\rho}}{d \rho}, \varepsilon_{\theta}=\frac{u_{\rho}}{\rho}, \quad \varepsilon_{\rho \theta}=\frac{d u_{\theta}}{d \rho}-\frac{u_{\theta}}{\rho} .
$$

Let $m^{*}$ be a certain fixed number of the reinforcement fiber classes. The Hook's law for a non-homogeneous reinforced material with $\mathrm{m}^{*}$ number of the reinforcement fiber classes is defined as $[8,10]$

$$
\begin{aligned}
& \sigma_{\rho}=\Omega \frac{E}{1-v^{2}}\left(\varepsilon_{\rho}+v \varepsilon_{\theta}\right)+\sum_{m=1}^{m^{*}} \sigma_{m} \omega_{m} \cos ^{2} \varphi_{m}, \\
& \sigma_{\theta}=\Omega \frac{E}{1-v^{2}}\left(\varepsilon_{\theta}+v \varepsilon_{\rho}\right)+\sum_{m=1}^{m^{*}} \sigma_{m} \omega_{m} \sin ^{2} \varphi_{m}, \\
& \sigma_{\rho \theta}=\Omega \frac{E}{1+v} \varepsilon_{\rho \theta}+\sum_{m=1}^{m^{*}} \sigma_{m} \omega_{m} \cos \varphi_{m} \sin \varphi_{m}, \Omega=1-\sum_{m=1}^{m^{*}} \omega_{m},
\end{aligned}
$$
material.

where E,v are the Young's modulus and the Poisson ratio, respectively, of the binding

If imposing additional conditions of the consistency of the fiber cross-sections [14], the reinforcement power $\omega_{m}$ by the $m$-th class of fibers meets the following conditions in the polar coordinate system

$$
\frac{\partial}{\partial \rho}\left(\rho \omega_{m} \cos \varphi_{m}\right)+\frac{\partial}{\partial \theta}\left(\omega_{m} \sin \varphi_{m}\right)=0
$$

In the problem under consideration we find the power $\omega_{m}$ from (5) after setting the equations for certain reinforcement trajectories $\rho=\rho(\theta)$, introducing the reinforcement angles $\varphi_{m}$ and initial conditions of the reinforcement yield. I integrate equation (5) taking into account the given reinforcement power $\omega_{0 m}$ on the inner contour $\rho=\rho_{1}$. The initial intensity of reinforcement by fiber families $\omega_{0 m}$ is determined by the technology of manufacturing the plate.

\section{Resolving the system of equations of the axisymmetric problem}

I formulate the problem of the axisymmetric deformation of the reinforced plate in shears $u_{\rho}, u_{\theta}$. For this purpose, I introduce relations (4) into equilibrium equations (2), after finding the stresses $\sigma_{m}$ in the fibers using the formulas

$$
\sigma_{m}=E_{m}\left(\varepsilon_{\rho} \cos ^{2} \varphi_{m}+\varepsilon_{\theta} \sin ^{2} \varphi_{m}+\varepsilon_{\rho \theta} \cos \varphi_{m} \sin \varphi_{m}\right)
$$

$E_{m}-$ is the Young's modulus of the fibers material.

The stresses $\sigma_{\rho}, \sigma_{\theta}, \sigma_{\rho \theta}$ with regard to the structural characterics will be the following

$$
\begin{aligned}
& \sigma_{\rho}=m_{1}\left(\varepsilon_{\rho}+v \varepsilon_{\theta}\right)+\sum_{m=1}^{m^{*}} E_{m} \omega_{m}\left(\varepsilon_{\rho} \cos ^{2} \varphi_{m}+\varepsilon_{\theta} \sin ^{2} \varphi_{m}+\varepsilon_{\rho \theta} \sin \varphi_{m} \cos \varphi_{m}\right) \cos ^{2} \varphi_{m}, \\
& \sigma_{\theta}=m_{1}\left(\varepsilon_{\rho}+v \varepsilon_{\theta}\right)+\sum_{m=1}^{m^{*}} E_{m} \omega_{m}\left(\varepsilon_{\rho} \cos ^{2} \varphi_{m}+\varepsilon_{\theta} \sin ^{2} \varphi_{m}+\varepsilon_{\rho \theta} \sin \varphi_{m} \cos \varphi_{m}\right) \sin ^{2} \varphi_{m}, \\
& \sigma_{\rho \theta}=m_{2} \varepsilon_{\rho \theta}+\sum_{m=1}^{m^{*}} E_{m} \omega_{m}\left(\varepsilon_{\rho} \cos ^{2} \varphi_{m}+\varepsilon_{\theta} \sin ^{2} \varphi_{m}+\varepsilon_{\rho \theta} \sin \varphi_{m} \cos \varphi_{m}\right) \sin \varphi_{m} \cos \varphi_{m} .
\end{aligned}
$$

I define the relations for the stresses $\sigma_{\rho}, \sigma_{\theta}, \sigma_{\rho \theta}$ as follows 


$$
\begin{aligned}
& \sigma_{\rho}=a_{11} \varepsilon_{\rho}+a_{12} \varepsilon_{\theta}+a_{13} \varepsilon_{\rho \theta}, \sigma_{\theta}=a_{12} \varepsilon_{\rho}+a_{22} \varepsilon_{\theta}+a_{23} \varepsilon_{\rho \theta}, \\
& \sigma_{\rho \theta}=a_{13} \varepsilon_{\rho}+a_{23} \varepsilon_{\theta}+a_{33} \varepsilon_{\rho \theta},\left(m_{1}=\Omega \frac{E}{1-v^{2}}, m_{2}=\Omega \frac{E}{1+v}\right),
\end{aligned}
$$

where the following coefficients are introduced

$$
\begin{aligned}
& a_{11}=m_{1}+\sum_{m=1}^{m^{*}} E_{m} \omega_{m} \cos ^{4} \varphi_{m}, a_{12}=v m_{1}+\sum_{m=1}^{m^{*}} E_{m} \omega_{m} \cos ^{2} \varphi_{m} \sin ^{2} \varphi_{m}, \\
& a_{13}=\sum_{m=1}^{m^{*}} E_{m} \omega_{m} \cos ^{3} \varphi_{m} \sin \varphi_{m}, a_{22}=m_{1}+\sum_{m=1}^{m^{*}} E_{m} \omega_{m} \sin ^{4} \varphi_{m}, \\
& a_{23}=\sum_{m=1}^{m^{*}} E_{m} \omega_{m} \cos \varphi_{m} \sin ^{3} \varphi_{m}, a_{33}=m_{2}+\sum_{m=1}^{m^{*}} E_{m} \omega_{m} \cos ^{2} \varphi_{m} \sin ^{2} \varphi_{m} .
\end{aligned}
$$

Having introduced (8) into equilibrium equations (2), taking into account (3), one obtains the following system of differential equations with regard to the shear components:

$$
\begin{aligned}
& a_{11} \frac{d^{2} u_{\rho}}{d \rho^{2}}+a_{13} \frac{d^{2} u_{\theta}}{d \rho^{2}}+\left(\frac{d a_{11}}{d \rho}+\frac{a_{11}}{\rho}\right) \frac{d u_{\rho}}{d \rho}+\left(-\frac{a_{23}}{\rho}+\frac{d a_{13}}{d \rho}\right) \frac{d u_{\theta}}{d \rho}+ \\
& +\left(\frac{1}{\rho} \frac{d a_{12}}{d \rho}-\frac{a_{22}}{\rho^{2}}\right) u_{\rho}+\left(-\frac{1}{\rho} \frac{d a_{13}}{d \rho}+\frac{a_{23}}{\rho^{2}}\right) u_{\theta}=0, \\
& a_{13} \frac{d^{2} u_{\rho}}{d \rho^{2}}+a_{33} \frac{d^{2} u_{\theta}}{d \rho^{2}}+\left(\frac{d a_{13}}{d \rho}+\frac{a_{23}}{\rho}+\frac{2 a_{13}}{\rho}\right) \frac{d u_{\rho}}{d \rho}+\left(\frac{d a_{33}}{d \rho}+\frac{a_{33}}{\rho}\right) \frac{d u_{\theta}}{d \rho}+ \\
& +\left(\frac{1}{\rho} \frac{d a_{23}}{d \rho}+\frac{a_{23}}{\rho^{2}}\right) u_{\rho}+\left(-\frac{1}{\rho} \frac{d a_{33}}{d \rho}-\frac{a_{33}}{\rho^{2}}\right) u_{\theta}=0 .
\end{aligned}
$$

I add four boundary conditions on the inner and outer contour of the circular plate to system (9). Let the following shears be set on the inner contour at $\rho=\rho_{1}$ :

a) $u_{\rho}=C_{1}^{*}, u_{\theta}=C_{2}^{*}$ (at $C_{1}^{*}=0, C_{2}^{*}=0$ - is a rigidly fixed shaft, at $C_{1}^{*}=0, C_{2}^{*} \neq 0$ the shaft twisting is possible).

b) on the outer contour $\rho=\rho_{2}$ radial and tangential forces $p_{n}, p_{\tau}$ are set. Taking into account relations (8) and (3), the conditions on the outer contour will be the following

$$
\begin{aligned}
& a_{11} \frac{d u_{\rho}}{d \rho}+a_{12} \frac{u_{\rho}}{\rho}+\left.a_{13}\left(\frac{d u_{\theta}}{d \rho}-\frac{u_{\theta}}{\rho}\right)\right|_{\rho=\rho_{2}}=p_{n}, \\
& a_{13} \frac{d u_{\rho}}{d \rho}+a_{23} \frac{u_{\rho}}{\rho}+\left.a_{33}\left(\frac{d u_{\theta}}{d \rho}-\frac{u_{\theta}}{\rho}\right)\right|_{\rho=\rho_{2}}=p_{\tau} .
\end{aligned}
$$

The following combinations are possible in the boundary conditions: a force and shear are set on the inner contour, while on the outer contour the remaining force and shear are set. System (9) and the boundary conditions a) and b) represent a generalized two-point boundary problem for the system of usual differential equations. The boundary conditions a) and b) for the general reinforcement case include both of the two unknown functions $u_{\rho}, u_{\theta}$, and their derivatives. The coefficients in the system contain a full set of the structural characteristics of the material: the number of the $\mathrm{m}^{*}$ classes of reinforcing fibers, mechanical characteristics of the binding material and fiber, the power $\omega_{m}$ and trigonometric functions of the reinforcement angles $\varphi_{m}$.

\section{The conditions of the reinforced material strength.}

The verification of the conditions of distroying an elastically-reinforced material has its own specific features. Let us briefly formulate the strength conditions of the reinforced material. Let the 
material of the isotropic binding have different ultimate strengths at tension $\sigma_{c}^{+}$and compression $\sigma_{c}^{-}$. In the case of a 2D - stress state, the strength condition of Balandin [15] for a nonhomogenuous material using the stresses $\sigma_{\rho}^{c}, \sigma_{\theta}^{c}, \sigma_{\rho \theta}^{c}$ in the binding for the polar coordinate system has the form [16]

$$
\left(\sigma_{\rho}^{c}\right)^{2}+\left(\sigma_{\theta}^{c}\right)^{2}-\sigma_{\rho}^{c} \sigma_{\theta}^{c}+3\left(\sigma_{\rho \theta}^{c}\right)^{2}+\left(\sigma_{c}^{-}-\sigma_{c}^{+}\right)\left(\sigma_{\rho}^{c}+\sigma_{\theta}^{c}\right)<\sigma_{c}^{+} \sigma_{c}^{-} .
$$

I denote $\left(\sigma_{\rho}^{c}\right)^{2}+\left(\sigma_{\theta}^{c}\right)^{2}-\sigma_{\rho}^{c} \sigma_{\theta}^{c}+3\left(\sigma_{\rho \theta}^{c}\right)^{2}+\left(\sigma_{c}^{-}-\sigma_{c}^{+}\right)\left(\sigma_{\rho}^{c}+\sigma_{\theta}^{c}\right)$ by $S$. I call $S$ a function of Balandin.

For the reinforcing fiber class, we assume the ultimate strength of the $m$-th fiber class at tension $\sigma_{m}^{+}$and compression $\sigma_{m}^{-}$to be different. The reinforcement fiber classes remain elastic if inequalities [16] are satisfied

$$
-\sigma_{m}^{-}<E_{m}\left(\varepsilon_{\rho} l_{1 m}^{2}+2 \varepsilon_{\rho \theta} l_{1 m} l_{2 m}+\varepsilon_{\theta} l_{2 m}^{2}\right)<\sigma_{m}^{+}
$$

\section{Numeric solution of the axisymmetric problem}

We create a numeric scheme with regard to the non- dimentional variables: the linear size of the plate is related to the outer radius, and in the stress relations all values are related to the maximum Young's modulus of the reinforcing fiber class.

System (9) and the formulated boundary conditions represent a generalized two-point boundary problem for the system of usual differential equations.

The coefficients in (9) contain a complete set of structural characteristics: the number of the reinforcing fiber classes, mechanical characteristics of the binding materials and fibers, the reinforcement power and trigonometric functions of the reinforcement angles.

For the numeric solution, non-dimensional system (9) is reduced to the system of four differential equations of the first order, then, a difference scheme is constructed which approximates the system of differential equations and boundary conditions with the second order accuracy. The obtained system of linear equations with the three-diagonal coefficient matrix is solved by the method of the orthogonal run [17].

In the numerical experiment I considered a circular plate with three types of the reinforcement structure: the reinforcement trajectories are the Archimedean and logarithmic spiral [18] classes (I) (Fig. 1 a)), the Archimedean spiral and "bicycle wheel spoke" classes (II) (Fig. 1 b)), the logarithmic spiral and isogonal (Fig. $1 \mathrm{c}$ )), the logarithmic spiral and "bicycle wheel spoke" classes (III) (Fig. 2). Aliminum is chosen as the binding material, with the reinforcement being performed with steel fibers. The mechanical characteristics of the metal-composite [19] and a set of variables for the construction are given in Table 1.
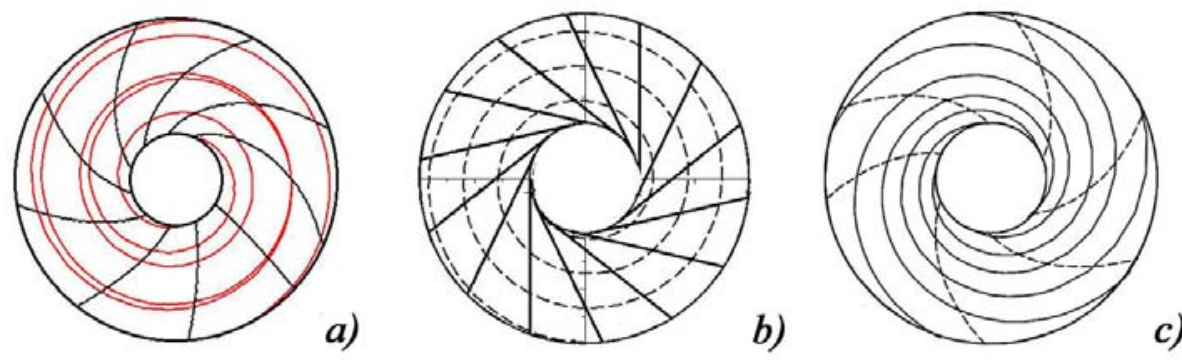

Fig. 1. Examples of plate reinforcement along curvilinear trajectories. 

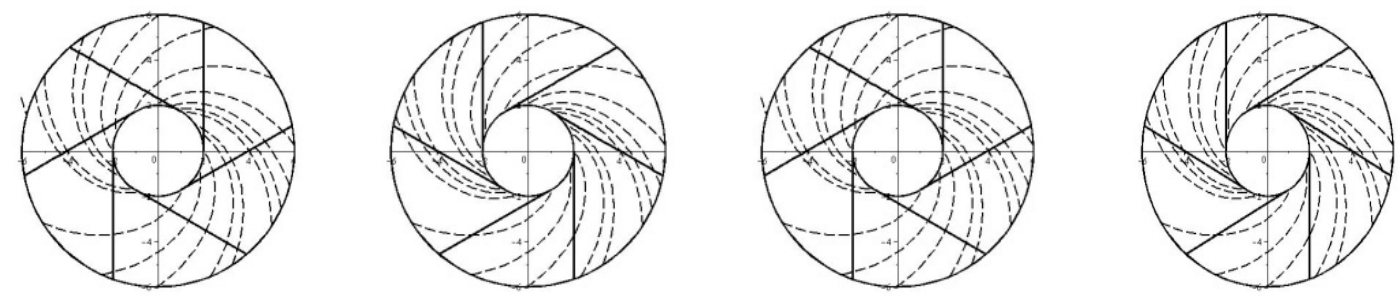

Fig. 2. Four structures of reinforcement along the bicycle wheel spoke trajectories and classes of the logarithmic spirals (clockwise and counterclockwise).

Table 1. Mechanical and geometical characteristics of the composite

\begin{tabular}{|l|l|l|l|l|l|l|}
\hline Amp & $v$ & $E, \mathrm{hPa}$ & $E_{1}, \mathrm{hPa}$ & $E_{2}, \mathrm{hPa}$ & $\omega_{01}$ & $\omega_{02}$ \\
\hline$-2,0$ & 0,3 & 70,0 & 200,0 & 200,0 & 0,3 & 0,3 \\
\hline$-1,5$ & 0,3 & 70,0 & 200,0 & 200,0 & 0,05 & 0,04 \\
\hline 0,5 & 0,3 & 70,0 & 200,0 & 200,0 & 0,32 & 0,1 \\
\hline 2,0 & 0,3 & 70,0 & 200,0 & 200,0 & 0,51 & 0,18 \\
\hline
\end{tabular}

The values of the average strength for the binding material $\sigma_{c}^{+}$amounts to $0,29 \mathrm{hPa}$. For the steel fibers, the ultimate strength at tension $\sigma_{1}^{+}=0,8 \mathrm{hPa}$, and compression $\sigma_{1}^{-}=0,4 \mathrm{hPa}$. The rigid embedment condition is set on the inner contour. The normal and tangent forces $p_{n}, p_{\tau}$ are set on the outer contour. In the boundary conditions, I assume $\frac{p_{n}}{p_{\tau}}=A m p$, and I take Amp to be the amplitude of the external loading, I assume the force value $p_{n}=1 \mathrm{hPa}$. Consider how the amplitude change influences the achievement of the ultimate load according to criteria (11), (12) for three reinforcement structures considered.

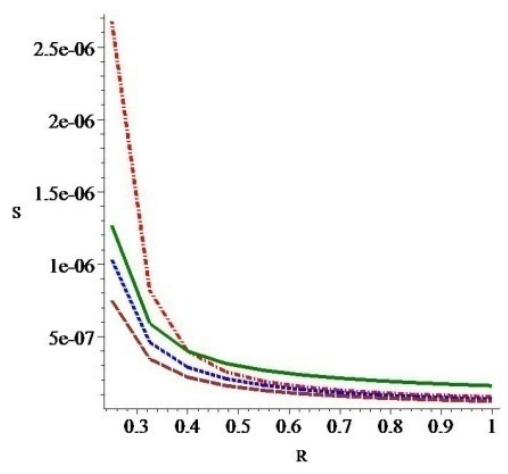

Fig. 3. Structure I.

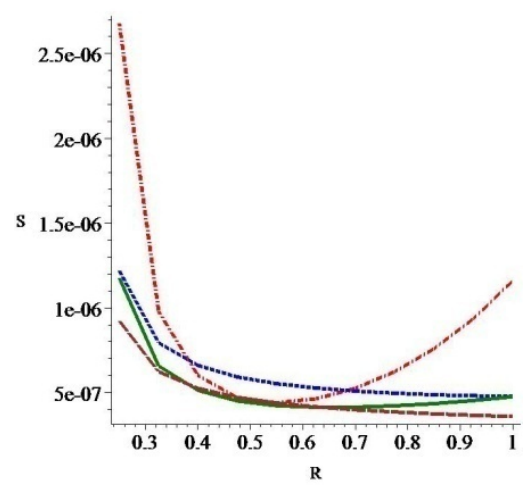

Fig. 4. Structure.

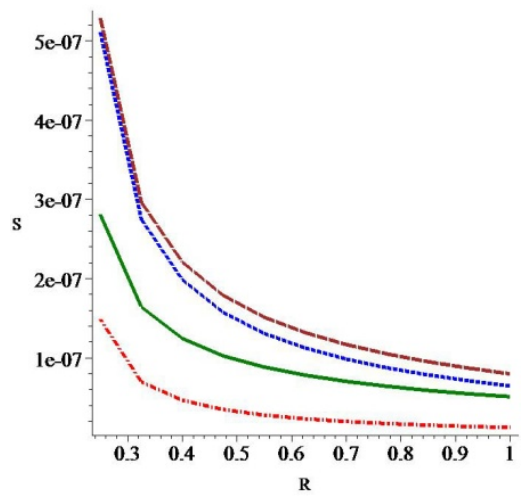

Fig. 5. Structure III.

Presented in Fig. 3 - Fig. 5 are the values of the Baladin function $S$ in non-dimentional variables $R \in[1 / 4,1]$ for four types of the initial reinforcement power $\omega_{01}, \omega_{02}$ from Table 1 at a fixed external load. As we can see, structure III allows one to significantly increase the external load for all the initial values of the reinforcement power as opposed to two other structures where the ultimate state is approached. One can conclude that at the given boundary conditions reinforcement structure III is the most preferable, with the material and loading level being fixed.

\section{Conclusions}


I derived a new curvilinear reinforcement of the plane structures. These novel kinds of reinforcement are logarithmic spiral classes, Archimedean spiral classes and the reinforcement along the bicycle. In addition, this study includes modelling the reinforcement along the trajectories which include the combinations of bicycle wheel spokes and the spiral classes mentioned above. The reinforcement along bicycle wheel spokes for a circular plate can have industrial applications. I demonstrate that the strength properties of the flat structure can be improved due to curvilinear reinforcement.

\section{References}

[1] V. V. Vasiliev, E. V. Morozov, Advanced Mechanics of Composite Materials, Elsevier, Oxford, Great Britain, 2007.

[2] N.A. Feodorova, Yu.V. Nemirovsky, Flat problem of the elastic environment reinforced with three families of fibres, Proceeding of the 9th International Symposium on Science and Technology, KORUS - 2005,1,1507769, 506-511.

[3] A.P Yankovakii, Deflections of uniformly stressed reinforced plates with account for their weakened resistance to the transverse shear, Journal of Applied Mechanics and Technical Physics, 58(1) (2017) 173-181.

[4] S.K. Golusko, Mathematical modeling and numerical calculation of composite strutures, Jornal of Physics: Conf. Series, 894 (2017).

[5] S.N. Astrakov, S.K. Golusko, L.A. Korolenko, Isoepiphanic shapes of high-pressure vessels, Journal of Applied and Indastrial Mathematics, 11 (2017) 305-311.

[6] Yu.V. Nemirovsky Yu.V. On the elastoplastic behaviour of reinforced layer, J. Appl. Mech. and Tech. Phys., 10 (6) (1969) 81-89.

[7] Yu.V Nemirovsky, V. D. Kurgusov, Strength and stiffness of concrete wall panels with complex reinforcement structures, News of Higher Educational Institutions, Construction, 2 (2003) 4-11.

[8] N.A. Feodorova, Yu.V. Nemirovsky, Modeling of deformed plane aerostructures reinforced by curvilinear fiber classes, Vestnik SibGAU, 2006. 6(13) (2006) 38-44.

[9] N. A. Feodorova ,Yu. V. Nemirovsky, Reinforecement of plane structures along orthogonal curvilinear trajectories, Vestn. Samar. Gos. Tekhn. Univ. Ser. Fiz.- Mat.Nauki 5(21) (2010) 96-104.

[10] N. A. Feodorova ,Yu. V. Nemirovsky, Mathematical modeling of the plane constructions from reinforced fibrous materials. Krasnoyarsk: Siberian Federal University, 2010.

[11] N.A. Feodorova, Modeling for reinforced with isogonal trajectories ring-shaped lamels in polar coordinate system, Journal of Siberian Federal University, 4(3) (2011) 400-405.

[12] N. A. Feodorova, M. P Galanin, Reinforcement of planar constructions along isogonal trajectories, J. Keldysh Institute preprints, 33 (2017) 1-16.

[13] Yu.V. Nemirovsky, On the elastic behavior of the reinforced layer, Int. J. Mech. Sci. 12 (1970) 898-903.

[14] S.B. Bushmanov, Yu.V Nemirovsky, Designing plates reinforced equally-stressed fibers with constant cross-section, Mech. Compos. Materials, 19 (2) (1983) 207-213.

[15] P.P. Balandin, On the question of strength hypotheses, Bulletin of engineers and technicians, 1 (1937) 19-24.

[16] Yu.V. Nemirovsky, B. S. Reznikov, Strength of structural elements from composite materials, Novosibirsk, Nauka, 1986.

[17] G. Ortega, W. Pool, An introduction into numerical methods of solving differential equations, New York, Pitman Publishing Ina., 1981.

[18] A.A.Savelov, Plane curves, Classification, features, application, Moscow, GIF-ML (State Publishing House on Physics and Mathematics Literature), 1960.

[19] V.V Vasilyev, V.D Protasov, et al., Composite materials, a handbook, Moscow, Mashinostroenie (Machine Muilding), 1990. 